\title{
Convexifying Monotone Polygons While Maintaining Internal Visibility
}

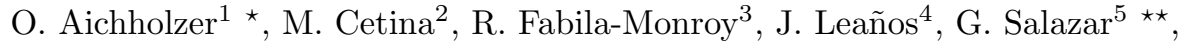 \\ and J. Urrutia ${ }^{6 \star \star \star}$ \\ 1 Institute for Software Technology, University of Technology, Graz, Austria. \\ oaich@ist.tugraz . at \\ 2 Instituto de Física, Universidad Autónoma de San Luis Potosí, México. \\ mcetina@gmail.com \\ 3 Departamento de Matemáticas, Cinvestav, México. \\ ruyfabila@math.cinvestav.edu.mx \\ 4 Unidad Académica de Matemáticas, Universidad Autónoma de Zacatecas, México. \\ jleanos@mate.reduaz.mx \\ 5 Instituto de Física, Universidad Autónoma de San Luis Potosí, México. \\ gsalazar@ifisica.uaslp.mx \\ ${ }^{6}$ Instituto de Matemáticas,Universidad Nacional Autónoma de México. \\ urrutia@matem. unam.mx
}

\begin{abstract}
Let $P$ be a simple polygon on the plane. Two vertices of $P$ are visible if the open line segment joining them is contained in the interior of $P$. In this paper we study the following questions posed in $[7,8]:(1)$ Is it true that every non-convex simple polygon has a vertex that can be continuously moved such that during the process no vertex-vertex visibility is lost and some vertex-vertex visibility is gained? (2) Can every simple polygon be convexified by continuously moving only one vertex at a time without losing any internal vertex-vertex visibility during the process?

We provide a counterexample to (1). We note that our counterexample uses a monotone polygon. We also show that question (2) has a positive answer for monotone polygons.
\end{abstract}

Keywords: convexification, monotone polygons, visibility graph

\section{Introduction}

Let $P$ be a simple polygon with vertices $\left\{p_{1}, \ldots, p_{n}\right\}$. We say that two vertices of $P$ are $P$-visible if the relative interior of the line segment joining them is contained in the interior of $P$. The visibility graph $V G(P)$ of $P$ is the graph with vertex set $\left\{p_{1}, \ldots, p_{n}\right\}$ in which two vertices of $P$ are adjacent if they are

\footnotetext{
* Partially supported by FWF [Austrian Fonds zur Förderung der Wissenschaftlichen Forschung] under grant S9205-N12, NFN Industrial Geometry.

** Supported by CONACYT Grant 106432.

$\star \star \star$ Partially supported by project SEP-CONACYT of México, Proyecto 80268.
} 
$P$-visible. A classical problem in computational geometry is that of convexifying simple polygons; that is, using a given fixed set of transformations that can be applied to the vertices and edges of $P$, try to transform $P$ into a convex polygon in such a way that some properties of $P$ are preserved. The first formulation of a problem of this kind was proposed by Erdös [4], who proposed a strategy to convexify a non-convex polygon by using flips; see also $[1,2,3,5,9,10]$.

Our starting point is the following question posed by Satyan L. Devadoss in the Open Problem Session at CCCG 2008 [7,8]:

Question 1. Given a simple polygon $P$ and its visibility graph $V G(P)$, can the vertices of $P$ be moved continuously (one at a time or simultaneously) along paths so that:

- the simplicity of the polygon $P$ is maintained all the time, and

- the visibility graph of $P$ never loses edges, only gains them.

In discussions following the workshop, the following two specific questions were raised [6]:

1. Is it true that every non-convex simple polygon has a vertex $p$ that can be continuously moved so that while $p$ moves, $V G(P)$ gains at least one extra edge, and never loses any?

2. Can every simple polygon be convexified by continuously moving only one vertex at a time such that $V G(P)$ never loses any edge?

Our aim is to prove that Question (2) has a positive answer for monotone polygons. On the other hand, we give an example that shows that the answer to Question (1) is negative, even for monotone polygons. For recent results on this topic, see also [8].

\subsection{Polygons and Visibility}

Let $\left\{p_{0}, \ldots, p_{n-1}\right\}$ be a set of points in the plane. A simple polygon $P$ with vertex set $\left\{p_{0}, \ldots, p_{n-1}\right\}$ is a non-crossing piecewise linear curve formed by the union of the closed line segments $\overline{p_{i} p_{i+1}}$ joining $p_{i}$ to $p_{i+1}, i=0, \ldots, n-1$, addition taken $\bmod n$.

A polygon $P$ is monotone if any vertical line intersects $P$ in at most two points. We will suppose without loss of generality that no vertical line passes through two vertices of $P$. Let $u$ and $v$ be the leftmost and rightmost vertices of $P$. Clearly there are two edge-disjoint paths contained in $P$ joining $u$ to $v$, one above the other. The first will be called the upper chain of $P$, the second the lower chain of $P$.

A basic operation that we will use in this paper is that of moving the elements of $\left\{p_{0}, \ldots, p_{n-1}\right\}$ around the plane. As the vertices of $P$ move, strictly speaking the polygon $P$ defined by its vertices changes, nevertheless, abusing our terminology a bit, we will always refer to it as $P$. In other words, as the 
elements of $\left\{p_{0}, \ldots, p_{n-1}\right\}$ move around the plane, they and $P$ keep their respective identities. We will restrict our point moves to those that do not destroy the simplicity of $P$.

In Figure 1(b), we illustrate the change that the polygon shown in Figure 1(a) undergoes as point $p$ moves. Moving $p$ from its initial position in Figure 1(a) to its position in Figure 1(c) is not allowed.

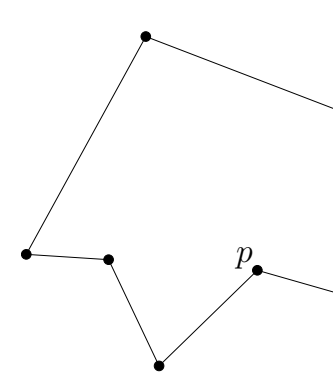

(a)

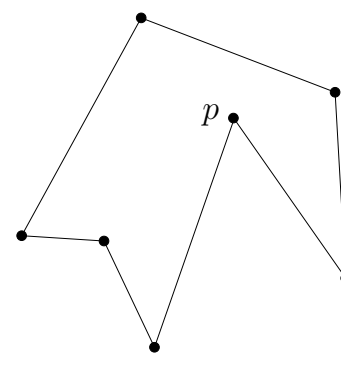

(b)

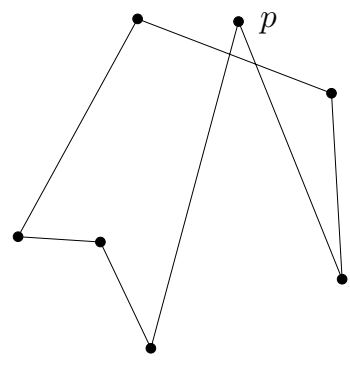

(c)

Fig. 1. (a) A polygon $P$. (b) A valid vertex move on $P$. (c) An invalid vertex move (simplicity is lost).

If $P$ is a polygon, then $\mathrm{CH}(P)$ denotes the convex hull of $P$. A vertex of $P$ that lies on the boundary of $\mathrm{CH}(P)$ is called a convex hull vertex, otherwise it is called an interior vertex. Let $V^{\bullet}(P)$ and $V^{\circ}(P)$ respectively denote the set of convex hull and interior vertices of $P$. Thus $V(P)=V^{\circ}(P) \cup V^{\bullet}(P)$.

A simple polygon $P$ divides mathbbR $R^{2} \backslash P$ into two regions, a bounded region called the interior of $P$, and an unbounded region called the exterior of $P$. We say the two vertices $u$ and $v$ of $P$ are $P$-visible if the relative interior of the line segment $\overline{u v}$ joining them is contained in the interior of $P$. We call $\{u, v\}$ a visibility pair. Note that according to our definition, consecutive vertices of $P$ are not visible. Let $\mathcal{N}(P)$ be the set of pairs of vertices of $P$ that are not $P$-visible. As consecutive vertices of $P$ are not $P$-visible, $|\mathcal{N}(P)| \geq n$.

The visibility graph $V G(P)$ is the graph with vertex set $\left\{p_{0}, \ldots, p_{n-1}\right\}$ in which two vertices are adjacent if they form a visibility pair. Note that if the vertices of $P$ move, the set of visible pairs of $P$ may change, and in turn $V G(P)$ may also change.

In this paper, we will be mainly concerned with the changes that polygons undergo as we move their vertices along line segments. Most of the time, we move one vertex at a time, and along a line segment. 
A point-move operation on $P$ is the translation of a vertex of $P$, say $p_{i}$, (the moving point) from an initial position on the plane $\left(x_{i}, y_{i}\right)$ to a final position $\left(x_{i}^{\prime}, y_{i}^{\prime}\right)$ along the line segment joining $\left(x_{i}, y_{i}\right)$ to $\left(x_{i}^{\prime}, y_{i}^{\prime}\right)$.

We say that a vertex move is visibility-preserving, if the following condition holds:

- If $p_{j}$ and $p_{k}$ were $P$-visible, they remain $P$-visible while $p_{i}$ moves.

If in addition the following is satisfied:

- The number of edges of $V G(P)$ increases,

we call it a visibility-increasing vertex move.

Our main results here are the following:

Theorem 1. There are polygons that have no visibility-increasing vertex moves.

and

Theorem 2. Every monotone polygon can be convexified with a sequence of visibility-preserving moves.

\section{A Counterexample to Question (1)}

Proof of Theorem 1. Consider the monotone polygon $P$ shown in Figure 2. The coordinates of the vertices of $P$ are $a=(-100,0), b=(-63,40), c=(-61,40)$, $d=(-33,2)$, and $e=(0,45)$. The points $\{f, g, h, i\}$ are obtained from the points $\{a, b, c, d\}$ by reflecting them along the $y$-axis. Points $b^{\prime}$ to $h^{\prime}$ are obtained from the points $b$ to $h$ by a reflection along the $x$-axis.

To show that $P$ does not admit any visibility-increasing vertex move, it is sufficient to consider the vertices of $P$ in the set $\left\{a, b, c^{\prime}, d, e\right\}$. The remaining cases follow by symmetry. For each of these vertices, we show in Figure 2 the open shaded region into which any of these points can be moved without losing any visibility pairs in $P$.

For example, the region $R$ into which vertex $a$ can move without destroying any visibility pair of $P$ is bounded by three lines; the one joining points $d$ and $f$, the line joining $d^{\prime}$ to $f^{\prime}$, and the line through points $b$ and $b^{\prime}$. If we move $a$ above the line joining $d$ to $f$, we lose visibility between $a$ and $f$. If we move $a$ to the right of the line joining $b$ and $b^{\prime}$, then the visibility between $b$ and $b^{\prime}$ is lost. Lastly, if we move $a$ below the line joining $d^{\prime}$ and $f^{\prime}$, we again lose visibility between $a$ and $f^{\prime}$. Moreover, it is easy to see that when we move $a$ around $R$, no additional internal visible pair of $P$ can be gained. A similar case analysis is easily done for the other vertices of $P$. This completes the proof of Theorem 1 . 


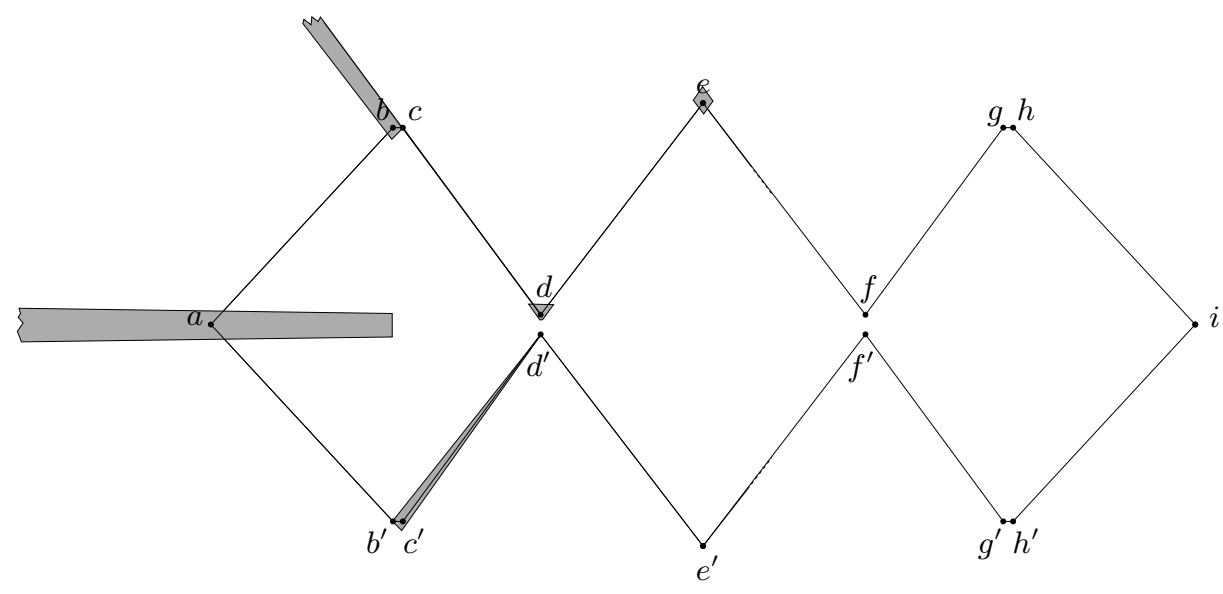

Fig. 2. A monotone polygon without visibility-increasing vertex moves. Shaded areas indicate visibility-preserving regions.

\section{$3 \quad$ Visibility-Preserving Vertex Moves}

In this section we establish some basic properties of visibility-preserving vertex moves. They will be used in the next section to prove our second main result. For a point $q \in \mathbb{R}^{2}$ and some $\delta>0$, we denote by $B_{\delta}(q)$ the closed disk with radius $\delta$ with center at point $q$.

Let $P=\left\{p_{0}, \ldots, p_{n-1}\right\}$ be a set of points on the plane in general position(no three collinear). We say that that $\delta>0$ is a safe threshold of $P$ if there are no three elements $p_{i}, p_{j}$, and $p_{k}$ of $P$ such that $B_{\delta}\left(p_{i}\right), B_{\delta}\left(p_{j}\right)$, and $B_{\delta}\left(p_{k}\right)$ are all intersected by a line. Equivalently, we can say that $\delta$ is a safe threshold of $P$ if there are no three points $p_{i}, p_{j}, p_{k} \in P$ such that when we translate each of them to a point within $\delta$ distance of them, they become aligned.

The following observations are evident, but useful.

Observation 1 Every point set $P$ in the plane in general position has a safe threshold $\delta$, for some $\delta>0$.

Observation 2 Let $P$ be a polygon such that $V(P)=\left\{p_{0}, \ldots, p_{n-1}\right\}$ is in general position. If a vertex move of a vertex $p_{i}$ of $P$ is not visibility-preserving, then at some point while $p_{i}$ is moving, it becomes collinear with two other vertices of $V(P)$.

The next proposition follows immediately from these two observations.

Proposition 1. Let $P$ be a polygon such that $V(P)$ is in general position, and let $\delta$ be a safe threshold of $V(P)$. Let $S=\left\{p_{\sigma(1)}, \ldots, p_{\sigma(m)}\right\}$ be any subset of vertices of $P$. Then if one at a time, each element $p_{\sigma(i)}$ of $S$ is moved from its original position to a new position within distance $\delta$ along a line segment, then every vertex move is visibility-preserving. 
The following lemma will be useful to prove Theorem 2 .

Lemma 1. Let $P$ be a monotone polygon. Then there is a sequence of visibilitypreserving vertex moves of some vertices of $P$ such that at the end of the sequence, the vertices of $P$ are in general position, $P$ remains monotone, and $\left|V^{\circ}(P)\right|+|\mathcal{N}(P)|$ does not increase during the vertex movements.

Proof. Recall that we are assuming that no vertical line passes through two vertices of $P$. We proceed by induction on the number of collinear triples in $V(P)$. Let tri(P) denote this number of collinear triples in $V(P)$. A vertex of $P$ is called weak if it is part of a collinear triple. If $\operatorname{tri}(\mathrm{P})=0$ there is nothing to prove. Observe now that for any $p_{i} \in P$, if we move it a small distance, say $\epsilon_{i}$, up or down along the vertical line through it, and in such a way that it does not cross any line joining two points of $P$, then:

- we do not create any new collinear triple, and

- if $p_{i}$ belongs to a collinear triple $p_{i}, p_{j}, p_{k}$, these points cease to form a collinear triple.

Suppose then that $\operatorname{tri}(\mathrm{P}) \geq 1$, and that a vertex $p_{i}$ of $P$ is part of one such triple. Assume without loss of generality that $p_{i}$ belongs to the upper chain of $P$ and that it is the leftmost vertex of the chain that participates in a collinear triple.

If $p$ is in $V^{\bullet}(P)$; that is, $p_{i}$ belongs to the convex hull of $P$, then move $p_{i}$ vertically down by a distance $\epsilon_{i}, \epsilon_{i}$ as described above, otherwise move $p_{i}$ vertically up by a distance $\epsilon_{i}$. In both cases tri(P) is reduced by at least one, and the sets $V^{\circ}(P)$ and $V^{\bullet}(P)$ remain unchanged. In the first case, $|\mathcal{N}(P)|$ might be reduced by one. Our result follows by induction on tri(P).

\section{Convexifying Monotone Polygons}

Proof of Theorem 2. By Lemma 1, we can assume that $V(P)$ is in general position. We proceed by induction on the sum of the number of interior vertices plus the number of non-visible pairs. If the vertices of $P$ are in convex position, there is nothing to prove. Observe that $P$ is convex if $\left|V^{\circ}(P)\right|+|\mathcal{N}(P)|=n$. Suppose then that $\left|V^{\circ}(P)\right|+|\mathcal{N}(P)|>n$ and assume that the theorem holds for all polygons $Q$ with $\left|V^{\circ}(Q)\right|+|\mathcal{N}(Q)|<\left|V^{\circ}(P)\right|+|\mathcal{N}(P)|$.

Since $P$ is not convex, it follows that $V^{\circ}(P)$ is not empty. Suppose without loss of generality that there are $k \geq 1$ interior vertices of $P$ on its upper chain. Relabel them as $v_{1}, v_{2}, \ldots, v_{k}$, in increasing order with respect to their $x$-coordinate.

Let $\delta>0$ be a safe threshold for the initial position of $V(P)$. Our algorithm will execute the following basic procedure $\mathbf{B P}$ once:

BP: One at a time from left to right, move $v_{1}, v_{2}, \ldots, v_{k}$ upwards, by a distance $\delta$.

Once $v_{1}, v_{2}, \ldots, v_{k}$ have all been moved, we execute $\mathbf{B P}$ repeatedly until one of the following occurs: 
1. a vertex in $\left\{v_{1}, v_{2}, \ldots, v_{k}\right\}$ reaches the convex hull of $P$,

2. a new visible pair occurs,

3. or the visibility-preserving property is lost.

If we stop because (1) or (2) occurs, then we are done, by our induction hypothesis. We will show now that (3) cannot happen, since before it happens there must be a visibility-increasing event. This will prove our result.

Observe that during the first iteration of $\mathbf{B P}$, we are moving $v_{1}, v_{2}, \ldots, v_{k}$ upwards by a distance $\delta$ which is the threshold of the initial configuration of $P$. Thus no collinear triple contained in $\left\{v_{1}, v_{2}, \ldots, v_{k}\right\}$ arises at any time during the first execution of BP. Observe also that at the end of each execution of $\mathbf{B P}$, if we consider only the elements of $\left\{v_{1}, v_{2}, \ldots, v_{k}\right\}, \delta$ is a safe threshold of them. In particular, this implies that if a visibility is lost during the execution of $\mathbf{B P}$, it must involve one or two elements of $P$ not in $\left\{v_{1}, v_{2}, \ldots, v_{k}\right\}$. We summarize this in the following observation:

Observation 3 During none of the next executions of $\boldsymbol{B P}$, is the visibility between any pair of points in $\left\{v_{1}, v_{2}, \ldots, v_{k}\right\}$ blocked by a third element in $\left\{v_{1}, v_{2}, \ldots, v_{k}\right\}$.

Suppose then that BP stops because (3) occurs before any event of type (1) or (2). Suppose that the critical move, i.e. the vertex move in which a visibility loss occurs for the first time when vertex $v_{i}, i \in\{1,2, \ldots, k\}$, is moved upwards during the $r$-th iteration of $\mathbf{B P}, r \geq 2$.

Thus before the critical move takes place, each of $v_{1}, v_{2}, \ldots, v_{i-1}$ has been moved upwards vertically $r \geq 2$ times, moving in total a distance $r \delta$. Each of $v_{i+1}, v_{i+2}, \ldots, v_{k}$ has been moved upwards vertically $(r-1)$ times.

Since during the critical move when $v_{i}$ moved from its second-to-last position to its last position a visibility of $P$ was lost, at some point in time $v_{i}$ passed over a point $p$ collinear with two vertices of $P$; call them $u$ and $w$, such that one of them, say $w$, blocked the visibility between $p$ and $u$; see Figure 3 .

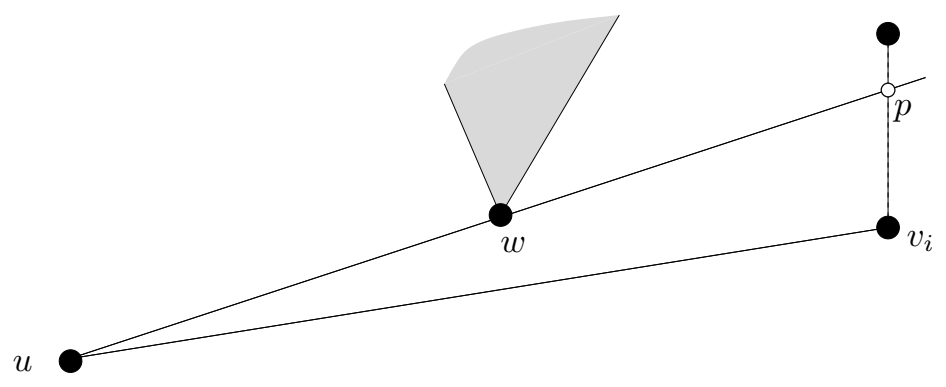

Fig. 3. When $v_{i}$ passes over $p, w$ blocks the visibility between $v_{i}$ and $u$. 
By Observation 3, at least one of $u$ or $w$ belongs to the convex hull of $P$. We now show that $w \in\left\{v_{1}, v_{2}, \ldots, v_{i-1}, v_{i+1}, \ldots, v_{k-1}, v_{k}\right\}$.

Obviously $w$ can not be in the lower chain of $P$. It remains to show that $w$ does not belong to $\mathrm{CH}(P)$. Let $p^{\prime}$ be the point where $v_{i}$ was located before the critical move was executed. Assume to the contrary that $w$ is in $\mathrm{CH}(P)$. Since before $v_{i}$ reached $p$, no event of type (1) took place, any point on the line segment joining $p$ to $p^{\prime}$ is in the interior of $\mathrm{CH}(P)$.

It is easy to see now that not only $w$, but also $p$ and $u$ must be in $\mathrm{CH}(P)$. Since $w$ is contained in the straight segment joining $p$ and $u$, and since $p$ is also in $\mathrm{CH}(P)$, it follows that there must be another vertex $z$ of $\mathrm{CH}(P)$ such that $p$ is contained in the straight line segment from $w$ to $z$. Thus $p, u, w$, and $z$ are all collinear. But $u, w$, and $z$ are distinct vertices of $\mathrm{CH}(P)$, contradicting that $V(P)$ is in general position. Thus $w=v_{j}$ for some $1 \leq j \leq k, j \neq i$ as claimed.

Since $w$ and $v_{i}$ belong to $\left\{v_{1}, v_{2}, \ldots, v_{k}\right\}$, then by Observation $3, u$ cannot belong to this set. Thus $u$ belongs to the lower chain of $P$ or to $\mathrm{CH}(P)$. To finish the proof it suffices to show that some vertex move performed before the so-called critical move was visibility-increasing.

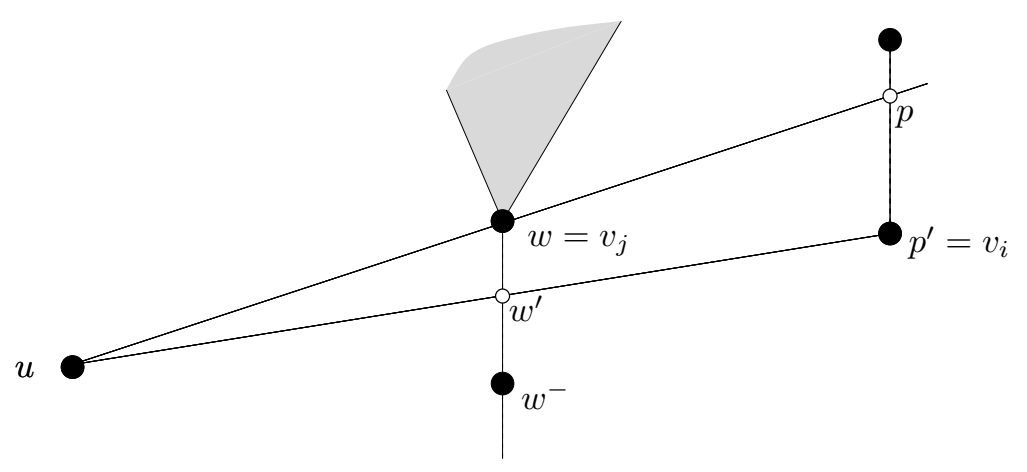

Fig. 4. In the polygon $P_{\lambda}, u$ is visible from $p^{\prime}=v_{i}$.

Consider the triangle whose vertices are $p, u$, and $p^{\prime}$; see Figure 4 . Let $L$ be the vertical line that passes through $w$, and let $w^{\prime}$ be the point where $L$ crosses $u p^{\prime}$. Since $p p^{\prime}$ has length $\leq \delta$, it follows that the length of the segment $w w^{\prime}$ is strictly less than $\delta$. Therefore the point $w^{-}$that lies a distance $\delta$ vertically below $w$ lies below $w^{\prime}$. Let $\lambda$ denote the vertex move that took $v_{j}$ from $w^{-}$to $w$, and let us denote by $P_{\lambda}$ the position of polygon $P$ before $\lambda$ was executed. We remark that $w^{-}$, and hence $\lambda$ and $P_{\lambda}$, are well defined, since every vertex was moved upwards at least once by a distance $\delta$ before any collinearity arose. 


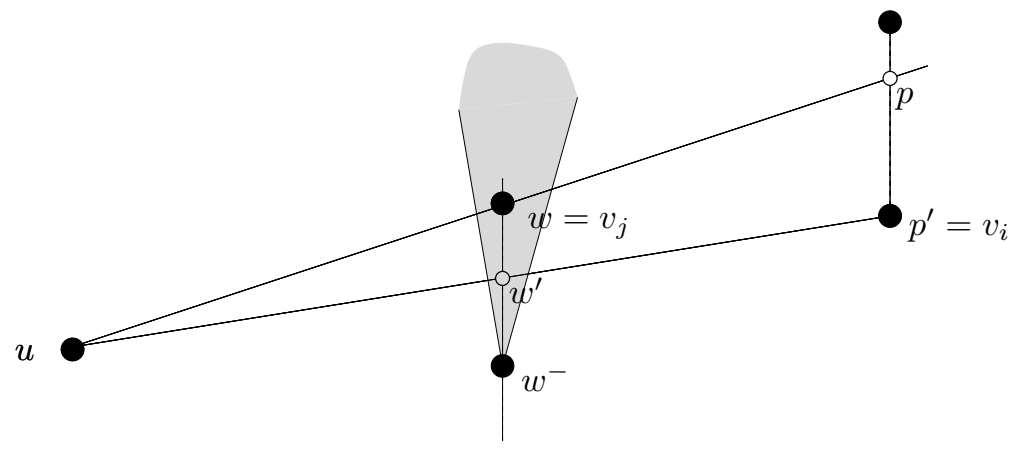

Fig. 5. In the polygon $P_{\lambda}, u$ is not visible from $p^{\prime}=v_{i}$. Therefore one of the moves involved in taking $P_{\lambda}$ into $P$ must be visibility-increasing.

The monotonicity of $P_{\lambda}$ implies that the open vertical ray above $w^{-}$is in the exterior of $P_{\lambda}$. Since this open vertical ray intersects $u p^{\prime}$ (namely at $w^{\prime}$ ), it follows that $u$ is not $P_{\lambda}$-visible from $p^{\prime}$; see Figure 5 . Since at the beginning of the critical move $u$ is $P$-visible from $p^{\prime}$, it follows that during one of the vertex moves executed between $\lambda$, call it $\lambda^{\prime}$, and the critical event, $v_{i}$ and $u$ became visible, thus $\lambda^{\prime}$ is visibility-increasing. Our result follows.

\section{Conclusion and Open Problems}

Although the proof of Theorem 2 is essentially algorithmic, it does not yield an efficient algorithm to convexify monotone polygons. In fact, the number of vertical vertex moves it requires can be arbitrarily large. For example for the polygon $P$ shown in Figure 6 , the number of steps required to convexify it depends on the distance between the line passing through vertices $r$ and $s$, and the line through $p$ and $q$. The closer these lines are, the more vertex moves we will have to execute to convexify $P$. The main problem is the size of the safe thresholds involved at each iteration of the main procedure. On the other hand, it is not hard to see that if we allow horizontal and vertical-vertex moves, then the polygon in Figure 6 can be convexified with a constant number of vertex moves. Can this problem be solved? We believe that this is not always possible. We conjecture:

Conjecture 1. There is a positive integer $n_{0}$ with the following property. For each integer $n \geq n_{0}$ and each $M$, there exists an $n$-vertex monotone polygon $P$ such that the number of visibility-preserving vertex moves required to convexify $P$ is at least $M$.

What happens if we allow more than one vertex to move at a time? In a $k$-vertex move, we allow up to $k$ vertices to move simultaneously. It is worth 


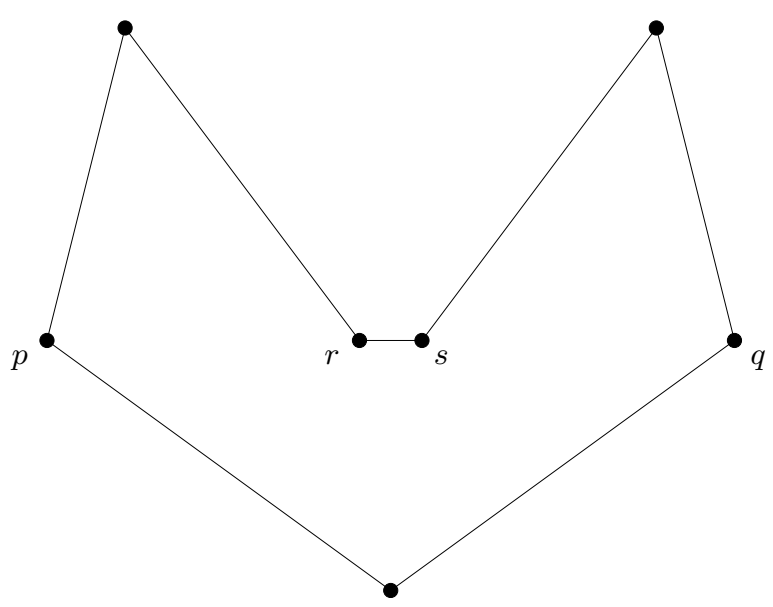

Fig. 6. The closer $\overline{r s}$ is to $\overline{p q}$, the more vertex moves are needed to convexify $P$.

mentioning that every monotone polygon on $n$ vertices can be convexified, preserving visibility, with only one $(n-3)$-vertex move. Indeed if all the vertices in the interior of $P$ in the lower (respectively upper) chain of $P$ move downwards (respectively upwards) towards $\mathrm{CH}(P)$ (each vertex stopping as soon as it reaches $\mathrm{CH}(\mathrm{P}))$, the resulting $(n-3)$-vertex move convexifies $P$, and is visibilitypreserving.

Thus the following question arises:

Question 2. Determine the complexity of the problem of convexifying a monotone polygon with $m$-vertex moves, $1 \leq m \leq n-3$.

What happens to Conjecture 1 and Question 2 if instead of monotone polygons, we consider simple polygons? Devadoss et al. [8] showed how to convexify star-shaped polygons by moving all the vertices in the polygon simultaneously. We believe that our techniques should extend to star-shaped polygons, but so far we have not been able to do it.

To conclude we mention that we believe that question (2) has a positive answer:

Conjecture 2. Every simple polygon can be convexified by a sequence of visibilitypreserving 1-vertex moves.

Acknowledgements. We would like to thank Erik Demaine and Stefan Langerman for valuable discusions on the topic.

\section{References}

1. G. Aloupis, B. Ballinger, P. Bose, M. Damian, E. D. Demaine, M. L. Demaine, R. Y. Flatland, F. Hurtado, S. Langerman, J. O'Rourke, P. Taslakian, and G. T. Tou- 
ssaint. Vertex Pops and Popturns. Proceedings of the 19th Canadian Conference on Computational Geometry (CCCG2007), pp. 137-140, 2007.

2. O. Aichholzer, C. Cortes, E. Demaine, V. Dujmovic, J. Erickson, H. Meijer, M. Overmars, B. Palop, S. Ramaswami, and G. Toussaint. Flipturning polygons. Discrete and Computational Geometry, Vol. 28, 2002, pp. 231-253.

3. T.C. Biedl, E. Demaine, S. Lazard, S. M. Robbins, and M.A. Soss. Convexifying monotone polygons. Lecture Notes in Computer Science 1741, 415424, 1999.

4. P. Erdős: Problem 3763. Amer. Math. Monthly 42 (1935), p.627. 463-470.

5. E. D. Demaine, B. Gassend, J. O'Rourke and G. T. Toussaint. All polygons flip finitely... right?. Surveys on discrete and computational geometry, Contemp. Math. Vol. 453, 2008, pp 231-255

6. E. D. Demaine, S. Langerman. Personal communication, Montreal, 2008.

7. E. D. Demaine, J. O'Rourkey. Open Problems from CCCG 2008. Proceedings of the 21st Canadian Conference on Computational Geometry (CCCG2009), pp. 75-78, 2009.

8. S.L. Devadoss, R. Shah, X. Shao, and E. Winston. Visibility graphs and deformations of associahedra. arXiv:0903.2848, March 2009.

9. B. Grünbaum. How to convexify a polygon. Geocombinatorics 5(1995), pp. 24-30.

10. B. de Sz.-Nagy. Solution of problem 3763. Amer. Math. Monthly 49(1939), pp. $176-177$. 